\title{
Mid-Infrared Imager, Spectrometer, Coronagraph (MISC) for the Origins Space Telescope (OST)
}

\author{
Itsuki Sakon, ${ }^{1}$ Thomas L. Roellig, ${ }^{2}$ Kimberly Ennico, ${ }^{2}$ Taro Matsuo,${ }^{3}$ Yuji Ikeda, ${ }^{4}$ \\ Tomoyasu Yamamuro, ${ }^{5}$ Naofumi Fujishiro, ${ }^{6}$ Keigo Enya, ${ }^{7}$ Olivier Guyon, ${ }^{8,9,10,11}$ Takayuki Kotani, ${ }^{8}$ \\ Jun Nishikawa, ${ }^{12}$ Yuki Sarugaku, ${ }^{13}$ Aor Takahashi, ${ }^{7}$ Takehiko Wada, ${ }^{7}$ Denis Burgarella,,${ }^{14}$ \\ OST/MISC TEAM, AND OST STDT
}

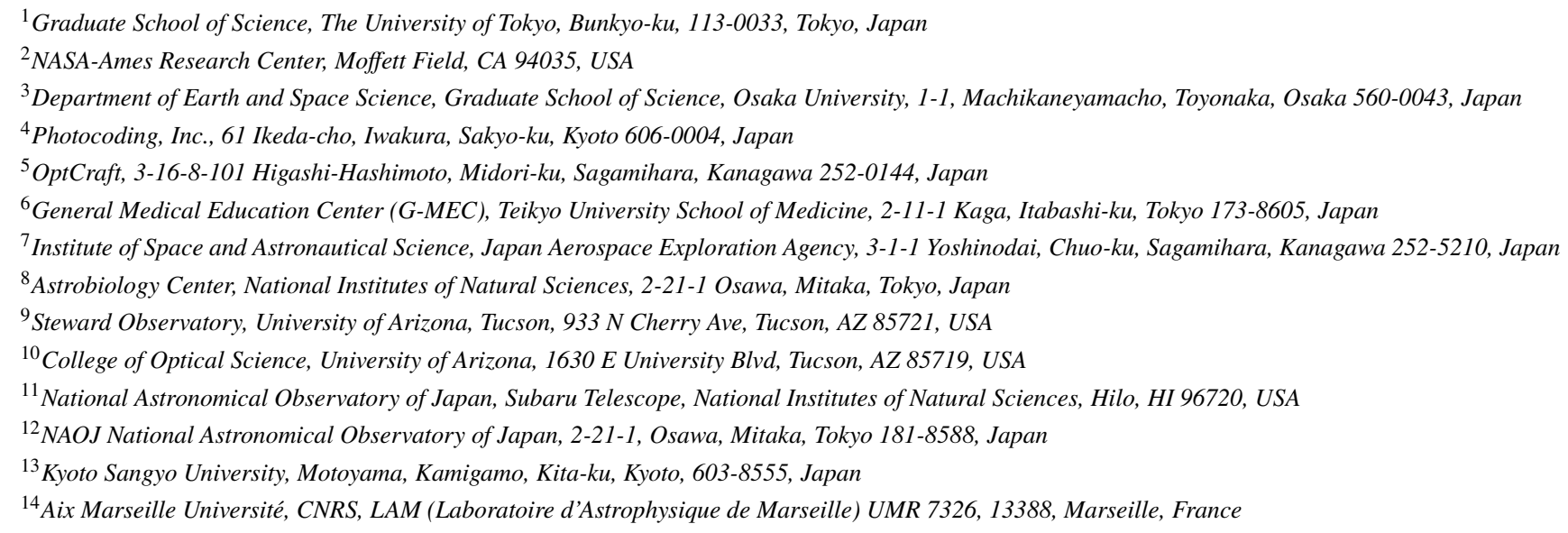

\begin{abstract}
The Origins Space Telescope (OST) is the mission concept for the Far-infrared Surveyor studied for the 2020 Astronomy and Astrophysics Decadal survey. Among the five instruments studied for the OST, JAXA is leading the study of the Mid-infrared Imager/Spectrometer/Coronagraph (MISC) with NASA/Ames in the framework of the community-based Science and Technology Definition Team (STDT) study. In order to provide the capability requested to address the science goals, MISC is designed to consist of three modules; 1) Mid-infrared Image and Spectrometer Module that covers the wavelengths in 6-38 $\mu \mathrm{m}$ with imaging and spectroscopy of low- to high-spectral resolution power, 2) Mid-infrared Coronagraph Module that covers the wavelengths in 6-38 $\mu \mathrm{m}$ and achieves $10^{-7}$ contrast at 0.5 arcsec from the central star and 3) Mid-infrared Transit Spectrometer Module that covers the wavelengths in 5-26 $\mu \mathrm{m}$ and achieves better than $10 \mathrm{ppm}$ stability on timescale of hours to days. In this proceedings, the design concept, the basic specification and the latest results of our optical designing study of MISC are shown.
\end{abstract}

Keywords: Origins Space Telescope, Future Mission

\section{OVERVIEW OF MID-INFRARED IMAGER/SPECTROMETER/CORONAGRAPH(MISC)}

\subsection{Science Traceability}

The Mid-infrared Imager/Spectrometer/Coronagraph (MISC) instrument will observe at the shortest wavelengths of any of the Origins Spece Telescope (OST; Meixner et al. 2016) instruments, ranging from 5 to $38 \mu \mathrm{m}$. The MISC instrument is designed to make measurements required for most of the top-rated Origins Space Telescope science programs. In particular, the science program "Bio-signatures of Transiting Exoplanets" aims to detect bio-signatures (e.g., ozone, water, and methane) in habitable worlds in both primary and secondary transits of exoplanets and requires spectroscopy in 5-18 $\mu \mathrm{m}(R \sim 100)$ and $17-25 \mu \mathrm{m}(R \sim 300)$ with very high spectro-photometric stability - better than $10 \mathrm{ppm}$ on timescales of hours to days. The science program "Direct Imaging of Exoplanets" aims to carry out the direct imaging and characterization of true exoplanet analogues of Jupiter and Saturn as well as ice giants at habitable temperatures 
$(\sim 300 \mathrm{~K})$ and requires coronagraph imaging and a low-resolution spectroscopic capability achieving $10^{-7}$ contrast at 0.5 $\operatorname{arcsec}(\sim 2 \times \lambda / D$ at $10 \mu \mathrm{m})$. MIR imaging capability in 5-40 $\mu \mathrm{m}$ is requested from the science programs "Bio-signatures of Transiting Exoplanets" and "Episodic Accretion in Protostellar Envelopes and Circumstellar Disks". Low-resolution ( $R \sim$ few hundreds) spectroscopic capability at 5-40 $\mu \mathrm{m}$ is required for the science programs "Connection Between Black Hole Growth and Star Formation Over Cosmic Time", "Star Formation and Multiphase ISM at Peak of Cosmic Star Formation", and "Birth of Galaxies During Cosmic Dark Ages". Medium-resolution (R few thousands) spectroscopic capability in 5-40 $\mathrm{m}$ is required for science programs "The Rise of Metals" and "Galaxy Feedback from SNe and AGN to $z \sim 3$ ". High-resolution ( $R \sim$ few tens thousands) spectroscopic capability at $10-40 \mu \mathrm{m}$ is required for science programs "Water Content of Planet-Forming Disks", "Direct Detection of Protoplanetary Disk Masses" and "Galaxy Feedback Mechanisms at $z<1$ ".

\subsection{Instrument Description}

The MISC consists of three separate optical modules providing imaging, spectroscopy, and coronagraph capabilities: The MISC Imager and Spectrometer Module (hereafter MISC I \& S) offers (1) a wide field imaging ( $3 \operatorname{arcmin} \times 3 \operatorname{arcmin})$ and low-resolution spectroscopic capability with filters and grisms for 6-38 $\mu \mathrm{m}$, (2) a medium-resolution $(R \sim 1,000)$ Integral Field Unit (IFU) spectroscopic capability for 9-38 microns (with a goal of 5-38 $\mu \mathrm{m}$ ) and (3) a high-resolution $(\mathrm{R} \sim 25,000)$ slit spectroscopic capability for 12-18 and 25-36 $\mu \mathrm{m}$. The MISC PIAA Coronagraph Module (hereafter MISC COR) covers 6-38 $\mu \mathrm{m}$. There is a special Densified Pupil Spectrometer Module (hereafter MISC TRA) that provides $R \sim 100-300$ exoplanet transit and emission spectroscopy from 6-26 $\mu \mathrm{m}$ with very high spectro-photometric stability. As the shortest wavelength focal plane imager the MISC I \& S Module will also be used for focal plane guiding as needed for the other OST science instruments as well as its own use. Tip-tilt mirrors and deformable mirrors in the MISC I \& S and COR modules improve pointing stability and wavefront performance beyond that provided by the OST telescope. The Figure 1 summarizes the basic measurement capabilities of the MISC instrument in the Concept 1 study.

\begin{tabular}{|c|c|c|c|c|c|}
\hline \multirow[t]{2}{*}{ Module } & \multicolumn{3}{|c|}{ MISC Imager \& Spectrometer } & \multirow{2}{*}{$\begin{array}{c}\text { MISC Transit Spectrometer } \\
\text { (Densified Pupil Spec.) } \\
\text { TRA-S/-M/-L }\end{array}$} & \multirow{2}{*}{$\begin{array}{c}\text { MISC Coronagraph } \\
\text { (PIAA) } \\
\text { COR-S } /-\mathrm{L}\end{array}$} \\
\hline & $\begin{array}{c}\text { Imager/Low-Res Spec. } \\
\text { WFI-S/-L }\end{array}$ & $\begin{array}{l}\text { Medium-Res Spec. } \\
\text { MRS-S/-M/-L* }\end{array}$ & $\begin{array}{c}\text { High-Res Spec. } \\
\text { HRS-S/-L }\end{array}$ & & \\
\hline Bandpass $(\mu \mathrm{m})$ & $6--38$ & $\begin{array}{c}10--36 \\
\text { (goal: } 5--36 \text { ) }\end{array}$ & $12--18,25--38$ & $5--26$ & $6--38$ \\
\hline $\begin{array}{l}\text { Spectral } \\
\text { Resolution }\end{array}$ & $\begin{array}{c}5-10 \text { [Imager }] \\
300[\text { Low-Res Spec. }]\end{array}$ & $1000-1500$ & $20,000-30,000$ & $\begin{array}{c}>100 \text { (TRA-S, TRA-M) } \\
300 \text { (TRA-L) }\end{array}$ & 300 \\
\hline Full FOV & $3 \operatorname{arcmin} \times 3 \operatorname{arcmin}$ [Imager] & $\begin{array}{c}3 \operatorname{arcsec} \times 5 \operatorname{arcsec} \\
\text { [with IFU] }\end{array}$ & & $3 \operatorname{arcsec} \times 3 \operatorname{arcsec}$ & $5.5 \operatorname{arcsec} \times 5.5 \operatorname{arcsec}$ \\
\hline $\begin{array}{c}\text { Slit for } \\
\text { Spectroscopy }\end{array}$ & $\begin{array}{c}\text { Length; } 3 \text { arcmin } \\
\text { Width; } 0.26 \text { arcsec (WFI-SG1) } \\
0.40 \text { arcsec (WFI-SG2) } \\
0.65 \text { arcsec (WFI-LG1) } \\
1.00 \text { arcsec (WFI-LG2) } \\
\text { [low-resolution Spec.] }\end{array}$ & $\begin{array}{c}\text { Length; } 3 \text { arcsec } \\
\text { (MRS-S/MRS-M/MRS-L) } \\
\text { Width; } 0.33 \text { arcsec (MRS-S) } \\
0.55 \text { arcsec (MRS-M) } \\
1.0 \text { arcsec (MRS-L) } \\
\text { \# of Slices; } 11 \text { (MRS-S) } \\
9 \text { (MRS-M), } 5 \text { (MRS-L) }\end{array}$ & \begin{tabular}{|c} 
Length; $1.0 \operatorname{arcsec}$ (HRS-S) \\
$2.0 \operatorname{arcsec}(H R S-L)$ \\
Width; $0.5 \operatorname{arcsec}(H R S-S)$ \\
$1.0 \operatorname{arcsec}(H R S-L)$
\end{tabular} & & $\begin{array}{c}\text { Length; } 1 \text { arcmin } \\
\text { Width; } 0.26 \text { arcsec (COR-SG1) } \\
0.40 \text { arcsec (COR-SG2) } \\
0.65 \text { arcsec (COR-LG1) } \\
1.00 \text { arcsec (COR-LG2) }\end{array}$ \\
\hline Detectors & $\begin{array}{l}2 \mathrm{kx} 2 \mathrm{k} \mathrm{Si}: \mathrm{As}(30 \mu \mathrm{m} / \mathrm{pix})[\mathrm{S}] \\
2 \mathrm{kx} 2 \mathrm{k} \mathrm{Si:Sb}(18 \mu \mathrm{m} / \mathrm{pix})[\mathrm{L}]\end{array}$ & $\begin{array}{l}\text { 2kx2k Si:As }(30 \mu \mathrm{m} / \mathrm{pix})[\mathrm{S}] \\
2 \mathrm{kx} 2 \mathrm{k} \mathrm{Si:As}(30 \mathrm{um} / \mathrm{pix})[\mathrm{M}] \\
1 \mathrm{kx} 1 \mathrm{k} \mathrm{Si}: \mathrm{Sb}(18 \mu \mathrm{m} / \mathrm{pix})[\mathrm{L}]\end{array}$ & $\begin{array}{l}2 \mathrm{k} \times 2 \mathrm{k} \mathrm{Si}: \mathrm{As}(30 \mu \mathrm{m} / \mathrm{pix})[\mathrm{S}] \\
1 \mathrm{k} \times 1 \mathrm{k} \mathrm{Si}: \mathrm{Sb}(18 \mu \mathrm{m} / \mathrm{pix})[\mathrm{L}]\end{array}$ & $\begin{array}{l}2 \mathrm{kx} 2 \mathrm{k} \text { Si:As }(30 \mu \mathrm{m} / \mathrm{pix})[\mathrm{S}] \\
2 \mathrm{k} \times 2 \mathrm{k} \text { Si:As }(30 \mu \mathrm{m} / \mathrm{pix})[\mathrm{M}] \\
2 \mathrm{k} \times 2 \mathrm{k} \text { Si:As }(30 \mathrm{~mm} / \mathrm{pix})[\mathrm{L}]\end{array}$ & $\begin{array}{l}\text { 2kx2k Si:As }(30 \mu \mathrm{m} / \mathrm{pix})[\mathrm{S}] \\
1 \mathrm{kx} 1 \mathrm{k} \mathrm{Si:Sb}(18 \mu \mathrm{m} / \mathrm{pix})[\mathrm{L}]\end{array}$ \\
\hline pixel scale & $0.088 \mathrm{arcsec} / \mathrm{pix}$ & $\begin{array}{c}0.0615 \mathrm{arcsec} / \mathrm{pix} \text { (MRS-S) } \\
0.10 \mathrm{arcsec} / \mathrm{pix} \text { (MRS-M) } \\
0.15 \mathrm{arcsec} / \mathrm{pix} \text { (MRS-L) }\end{array}$ & $\begin{array}{l}0.17 \mathrm{arcsec} / \mathrm{pix}[\mathrm{S}] \\
0.34 \mathrm{arcsec} / \mathrm{pix}[\mathrm{L}]\end{array}$ & $0.1 \mathrm{arcsec} / \mathrm{pix}$ & $\begin{array}{l}0.05 \mathrm{arcsec} / \mathrm{pix}(\mathrm{COR}-\mathrm{S}) \\
0.10 \mathrm{arcsec} / \mathrm{pix}(\mathrm{COR}-\mathrm{L})\end{array}$ \\
\hline $\begin{array}{l}\text { Specification } \\
\text { (Sensitivity/ } \\
\text { Stability/ } \\
\text { Contrast) }\end{array}$ & 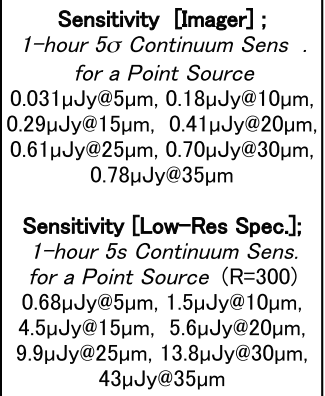 & $\begin{array}{c}\text { Sensitivity; } \\
\text { 1-hour 5s Continuum Sens. } \\
\text { for a Point Source }\left(R^{\sim} 1200\right) \\
3.4 \mu J y @ 7 \mu \mathrm{m}, 11 \mu \mathrm{J} @ 15 \mu \mathrm{m}, \\
34 \mu J y @ 24 \mu \mathrm{m}, 114 \mu J y @ 32 \mu \mathrm{m} \\
\\
\text { 1-hour 5s Line Sens. } \\
\text { for a Point Source } \\
1.1 \times 10^{-21} \mathrm{~W} / \mathrm{m}^{2} @ 7 \mu \mathrm{m}, \\
2.3 \times 10^{-21} \mathrm{~W} / \mathrm{m}^{2} @ 15 \mu \mathrm{m}, \\
3.4 \times 10^{-21} \mathrm{~W} / \mathrm{m}^{2} @ 24 \mu \mathrm{m}, \\
1.1 \times 10^{-20} \mathrm{~W} / \mathrm{m}^{2} @ 32 \mu \mathrm{m}\end{array}$ & $\begin{array}{c}\text { Sensitivity; } \\
\text { 1-hour } 5 \text { s Line Sens. } \\
\text { for a Point Source } \\
1.2 \times 10^{-21} \mathrm{~W} / \mathrm{m}^{2} @ 15 \mu \mathrm{m}, \\
3.6 \times 10^{-21} \mathrm{~W} / \mathrm{m}^{2} @ 30 \mu \mathrm{m}\end{array}$ & $\begin{array}{c}\text { Photometric stability; } \\
3-5 \text { ppm on timescales of } \\
\text { hours to days } \\
\text { (excluding the fluctuation of } \\
\text { detector gain) }\end{array}$ & $\begin{array}{c}\text { Average contrast; } \\
7 \times 10^{-6} \text { for } 10 \% \text { band } \\
1 \times 10^{-6} \text { for } 4 \% \text { band } \\
\text { in } 0.88-3.6 \lambda / D\end{array}$ \\
\hline
\end{tabular}

Figure 1. The MISC Fact Sheet

As a result of a trade-off study on coronagraphy methods among: a PIAA coronagraph (Guyon et al. 2014); a 4QPM coronagraph (e.g., Lajoie et al. 2014); a 8OPM coronagraph (Murakami et al. 2016); a Vortex coronagraph (Mawet et al. 2011); and a binary pupil mask (BPM) coronagraph (Enya \& Abe 2010), we have chosen the PIAA coronagraph as the baseline concept for the MISC COR. Although the PIAA coronagraph method requires its own independent module 
within the MISC instrument, it achieves the highest contrast at a small inner working angles with the highest throughput among the various types of coronagraphy methods. The 4QPM and 8OPM methods have the advantage that they do not necessarily require an independent module and that they can add a coronagraphic capability to MISC I \& S module with a relatively minor impact on its optical and mechanical design. However, as is the case for Vortex coronagraph, they require very stringent pointing accuracy and pointing stability requirements (i.e., a pointing jitter of $0.04 \times \lambda / D$ generates a $10^{-7}$-level leak at $2 \times \lambda / D$ and, therefore, a pointing accuracy and pointing stability better than 4 mas is requested) and, above all, the fabrication of the phase masks that achieve a homogeneous capability in a wide mid-infrared wavelength range requires further technical development.

A densified pupil spectroscopy is a newly-studied method for transit spectroscopy (Matsuo et al. 2016). This method will greatly improve spectro-photometric accuracy performance against optical disturbances. The science image is not disturbed by minor telescope pointing jitter or deformation of primary mirror. A large number of science pixels minimizes intra- and inter-pixel sensitivity variations. A number of reference pixels are also employed for calibration of potential detector gain fluctuations with a cold photon shield mask. An optimal calibration technique using the reference pixels will be developed using a test bed of a prototype system during development.

\section{THE OPTICAL AND MECHANICAL DESIGN OF THE MISC INSTRUMENT}

Based on the latest results of the optical and mechanical design of the MISC instrument, the 3D solid models of the MISC I \& S module, the MISC COR module and the MISC TRA module are shown in Figure 2.

Since the telescope optics is limited to image quality diffraction-limited performance at $20 \mu \mathrm{m}$ and longer wavelengths, the MISC needs to have its own internal wave front error correction optics in two of its modules. The MISC I \& S includes a DM and a TTM in its fore-optics and achieves diffraction limited image performance at $>5 \mu \mathrm{m}$ for sources within a wide 3 arcmin $\times 3$ arcmin FOV. The MISC COR includes a DM and a TTM in its fore-optics to correct for the telescope's wave front errors. The DM assembly with 32 × 32 actuators is used to exclude the speckles in regions up to $16 \times \lambda / D$ from the PSF peak (Takahashi et al. 2017).

\section{SUMMARY}

The MISC consists of the MISC I \& S module, the MISC COR module and the MISC TRA module. The MISC I $\& \mathrm{~S}$ offers (1) a wide field imaging (3 arcmin $\times 3$ arcmin) and low-resolution spectroscopic capability with filters and grisms for 6-38 $\mu \mathrm{m},(2)$ a medium-resolution $(R \sim 1,000)$ Integral Field Unit (IFU) spectroscopic capability for 5-38 $\mu \mathrm{m}$ and (3) a high-resolution $(R \sim 25,000)$ slit spectroscopic capability for $12-18$ and $25-36 \mu \mathrm{m}$. The MISC COR module employs PIAA coronagraphy method and covers 6-38 $\mu \mathrm{m}$ achieving $10^{-7}$ contrast at 0.5 arcsec from the central star. The MISC TRA module employs a densified pupil spectroscopic design and covers 5-26 $\mu \mathrm{m}$ by achieving 3-5 ppm of spectro-photometric stability. The highest ever spectro-photometric stability achieved by MISC TRA module as well as the highest-contrast coronagraphic imaging and spectroscopic capability at small inner working angle from the central bright source achieved by MISC COR module makes the OST a powerful tool to bring an revolutionary progress in exoplanet sciences. Combined with the spectroscopic capability in the FIR provided particularly by MRSS, the MISC widens the wavelength coverage of $O S T$ down to $5 \mu \mathrm{m}$, which makes the $O S T$ a powerful tool to diagnose the physical and chemical condition of the ISM using dust features, molecules lines and atomic and ionic lines. The MISC I \& S imager provides the $O S T$ a focal plane guiding function for the other $O S T$ science instruments as well as its own use.

In the Concept 1 study, JAXA is leading the study of the MISC instrument with NASA Ames Research Center (NASA/ARC). Laboratoire d'Astrophysique de Marseille is making a contribution to the study of the MISC instrument from both science and technical aspects. The wavelength coverage and the capabilities of the MISC instrument are partly overlapping with those of the AKARI/IRC (Onaka et al. 2007), Spitzer IRS (Houck et al. 2004), JWST/MIRI (Rieke et al. 2015; Wright et al. 2015), SOFIA/FORCAST (Adams et al. 2010), SOFIA/EXES (Richter et al. 2010; DeWitt et al. 2012), SPICA/MCS (Kataza et al. 2012), SPICA/SCI (Enya et al. 2008), SPICA/SMI (Kaneda et al. 2016; Sakon et al. 2016), TAO/MIMIZUKU (Miyata et al. 2010) and TMT/MICHI (Packham et al. 2012) instruments. Further international partnership makes it more efficient to use those heritages of space-qualified components in other space IR missions and is desirable to complete the technical challenges that have low TRL at present. Further information on the MISC instrument is available at http://exoplanets.astron.s.u-tokyo.ac.jp/OST/MISC/index_misc_case_A.html.

\section{REFERENCES}

Adams, J. D., Herter, T. L., Gull, G. E., et al. 2010, Proc. SPIE, 7735, 77351U

DeWitt, C., Richter, M. J., McKelvey, M. E., et al. 2012, Proc. SPIE, 8446, 84461A

Enya, K., Abe, L., Haze, K., et al. 2008, Proc. SPIE, 7010, 70102Z

Enya, K., \& Abe, L. 2010, PASJ, 62, 1407 


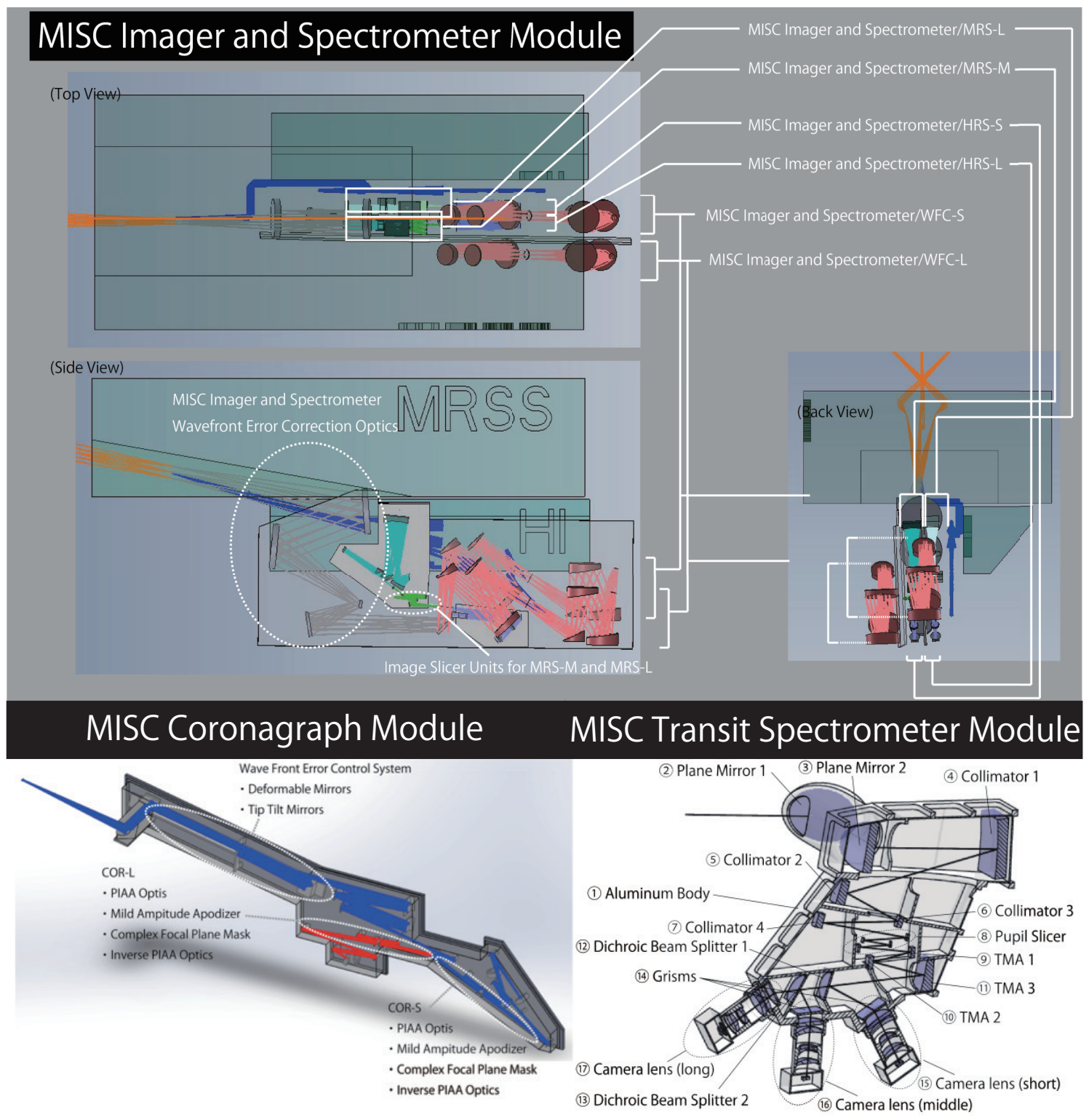

Figure 2. The 3D sold model of the MISC I\& S module, MISC COR module and MISC TRA module.

Guyon, O., Hinz, P. M., Cady, E., Belikov, R., \& Martinache, F. 2014, ApJ, 780, 171

Houck, J. R., Roellig, T. L., van Cleve, J., et al. 2004, ApJS, 154, 18

Kaneda, H., Ishihara, D., Oyabu, S., et al. 2016, Proc. SPIE, 9904, 99042I

Kataza, H., Wada, T., Sakon, I., et al. 2012, Proc. SPIE, 8442, 84420Q

Lajoie, C.-P., Soummer, R., Hines, D. C., \& Rieke, G. H. 2014, Proc. SPIE, 9143, 91433R

Matsuo, T., Itoh, S., Shibai, H., Sumi, T., \& Yamamuro, T. 2016, ApJ, 823, 139

Mawet, D., Murakami, N., Delacroix, C., et al. 2011, Proc. SPIE, 8151, 815108

Meixner, M., Cooray, A., Carter, R., et al. 2016, Proc. SPIE, 9904, 99040K

Miyata, T., Sako, S., Nakamura, T., et al. 2010, Proc. SPIE, 7735, 77353P

Murakami, N., Nishikawa, J., Akaiwa, N., et al. 2016, Proc. SPIE, 9912, $99126 \mathrm{G}$

Onaka, T., Matsuhara, H., Wada, T., et al. 2007, PASJ, 59, S401

Packham, C., Honda, M., Richter, M., et al. 2012, Proc. SPIE, 8446, 84467G

Richter, M. J., Ennico, K. A., McKelvey, M. E., \& Seifahrt, A. 2010, Proc. SPIE, 7735, 77356Q

Rieke, G. H., Wright, G. S., Böker, T., et al. 2015, PASP, 127, 584

Sakon, I., Kaneda, H., Oyabu, S., et al. 2016, Proc. SPIE, 9904, 99043V

Takahashi, A., Enya, K., Haze, K., et al. 2017, Applied Optics, Vol.56, Issue 23, 6694-6708

Wright, G. S., Wright, D., Goodson, G. B., et al. 2015, PASP, 127, 595 